\title{
XMM-Newton observation of the persistent Be/NS X-ray binary pulsar RX J1037.5-5647 in a low luminosity state
}

\author{
N. La Palombara ${ }^{1}$, L. Sidoli ${ }^{1}$, P. Esposito ${ }^{1,2}$, A. Tiengo ${ }^{1}$, and S. Mereghetti ${ }^{1}$ \\ 1 INAF, Istituto di Astrofisica Spaziale e Fisica Cosmica - Milano, via Bassini 15, 20133, Milano, Italy \\ e-mail: nicola@iasf-milano.inaf.it \\ 2 INFN, Sezione di Pavia, via A.Bassi 6, 27100, Pavia, Italy \\ Received 20 May 2009 / Accepted 16 July 2009
}

ABSTRACT

\begin{abstract}
The spectra of several X-ray binary pulsars display a clear soft excess, which in most cases can be described with a blackbody model, above the main power-law component. While in the high-luminosity sources it is usually characterized by low temperature $(k T<0.5 \mathrm{keV})$ and a large emission radius $(R>100 \mathrm{~km})$, in the two persistent and low-luminosity pulsars $4 \mathrm{U} 0352+309$ and RX J0146.9+6121 this component has a high temperature $(k T>1 \mathrm{keV})$ and a smaller radius $(R<0.5 \mathrm{~km})$, consistent with the estimated size of the neutron-star polar cap. Here we report on the timing and spectral analysis of RX J1037.5-5647, another lowluminosity persistent Be binary pulsar, based on the first XMM-Newton observation of this source. We have found a best-fit period $P=853.4 \pm 0.2 \mathrm{~s}$, which implies an average pulsar spin-up $\dot{P} \simeq-2 \times 10^{-8} \mathrm{~s} \mathrm{~s}^{-1}$ in the last decade. The estimated source luminosity is $L_{\mathrm{X}} \sim 10^{34} \mathrm{erg} \mathrm{s}^{-1}$, a value comparable to the other persistent Be binary pulsars and about one order of magnitude lower than in most of the previous measurements. The source spectrum can be described with a power law plus blackbody model, with $k T_{\mathrm{BB}}=1.26_{-0.09}^{+0.16} \mathrm{keV}$ and $R_{\mathrm{BB}}=128_{-21}^{+13} \mathrm{~m}$, suggesting a polar-cap origin of this component. These results strengthen the hypothesis that, in addition to low luminosities and long periods, this class of sources is also characterized by common spectral properties.
\end{abstract}

Key words. X-rays: binaries - accretion, accretion disks - stars: emission-line, Be - stars: pulsars: individual: LS 1698 X-rays: individuals: RX J1037.5-5647

\section{Introduction}

Most X-ray binary pulsars (XBPs) are high mass X-ray binaries (HMXRBs) in which a neutron star (NS) with a magnetic field $B \sim 10^{12} \mathrm{G}$ accretes matter from a high-mass early-type star, either an OB supergiant or a Be star. The X-ray spectra of both subgroups are generally described by a rather flat power law between 0.1 and $10 \mathrm{keV}$ (photon index $\sim 1$ ) with a high-energy cutoff (Nagase 2002). Several of the brightest XBPs $\left(L_{\mathrm{X}} \sim 10^{37-38} \mathrm{erg} \mathrm{s}^{-1}\right)$ also have a marked soft X-ray excess (Ramsay et al. 2002; Paul et al. 2002; Naik \& Paul 2004; Yokogawa et al. 2000b; Kohno et al. 2000; Burderi et al. 2000; Manousakis et al. 2009). A variety of simple models (both thermal and non thermal) have been proposed to fit this feature. Only in a few cases does this low-energy component unambiguously show coherent pulses, so the debate over its origin remains open.

A large number of new pulsars in BeXRB systems have been discovered in the Small Magellanic Cloud, where the observations are unaffected by the high interstellar absorption along the Galactic plane (Haberl \& Sasaki 2000; Israel et al. 2000; Yokogawa et al. 2003; Macomb et al. 2003; Haberl et al. 2008; Eger \& Haberl 2008). This allowed detailed study of their X-ray spectra down to energies of a few hundred $\mathrm{eV}$, leading to detection of a marked soft excess in most pulsars, also in sources with relatively low luminosity $\left(L_{\mathrm{X}} \sim 10^{35-36} \mathrm{erg} \mathrm{s}^{-1}\right.$ ) (Yokogawa et al. 2000a; Sasaki et al. 2003; Haberl \& Pietsch 2004; Ueno et al. 2004; Majid et al. 2004; Haberl \& Pietsch 2005).

Based on these results, Hickox et al. (2004) show that a lowenergy component should be visible in all X-ray pulsars that have sufficiently high flux and small absorption. In fact, most of the soft-excess sources are at small distances and/or away from the Galactic plane (most of them are in the Magellanic
Clouds). This suggests that the presence of a soft spectral component could be a very common, if not a ubiquitous, feature intrinsic to X-ray pulsars. Different explanations for its origin have been proposed, depending on the source luminosity. When $L_{X} \geq$ $10^{38} \mathrm{erg} \mathrm{s}^{-1}$, the luminosity and the shape of the soft component can only be explained by an optically-thick accretion disk, which reprocesses the hard X-rays coming from the neutron star at its inner edge. In less luminous sources, with $L_{X} \leq 10^{36} \mathrm{erg} \mathrm{s}^{-1}$, the soft excess can come from other processes, such as emission by photo-ionized or collisionally-heated diffuse gas or thermal emission from the surface of the neutron star. Finally, in the sources of intermediate luminosity, either or both of these types of emission can be present.

Recently, a clear thermal excess has also been observed in the cases of RX J0146.9+6121 (La Palombara \& Mereghetti 2006) and 4U 0352+309 (La Palombara \& Mereghetti 2007), which are two persistent Be pulsars. This is a specific class of binary pulsars characterized by low luminosity $\left(L_{\mathrm{X}} \sim\right.$ $\left.10^{34-35} \mathrm{erg} \mathrm{s}^{-1}\right)$ and a long pulse period $(P>100 \mathrm{~s})$; moreover, they show no transient behavior, since the source luminosity varies at most by a factor $\sim 10$ (Negueruela 1998). These properties suggest that the Be star orbits the NS in a wide and nearly circular orbit, continuously accreting material from the low-density outer regions of the circumstellar envelope. This picture has been confirmed for 4U $0352+309$ by the discovery of an orbital period of 250.3 days (Delgado-Martí et al. 2001). The detection of the thermal excess in these two persistent pulsars was favored by their small distance $(d \leq 2.5 \mathrm{kpc})$ and interstellar absorption $\left(N_{\mathrm{H}} \sim 10^{21} \mathrm{~cm}^{-2}\right)$.

It is interesting to investigate whether other persistent Be binary pulsars are characterized by the same type of spectral feature, in order to check that it is a common property of this class 
of sources. To this aim, we observed the Be/NS binary pulsar RX J1037.5-5647 with XMM-Newton, identified with LS 1698, a B0 III-Ve star at $\sim 5 \mathrm{kpc}$ (Motch et al. 1997). This source is most likely associated to the UHURU source 4U 1036-56 (Forman et al. 1978), the ARIEL $V$ source 3A 1036-565 (Warwick et al. 1981), and the OSO 7 source 1M 1022-554 (Markert et al. 1979). Thanks to observations performed by the $P C A$ instrument on board RossiXTE, Reig \& Roche (1999) performed the first detailed timing and spectral analysis, and discovered a pulsation with period $P=860 \pm 2$ s. The source spectrum could be described by a cut-off power law, with a low cut-off energy $\left(E_{\text {cut }}=4.7 \mathrm{keV}\right)$ and a weak (equivalent width $\left.\mathrm{EQW} \simeq 65 \mathrm{eV}\right)$ iron line at $6.5 \mathrm{keV}$. Moreover, the estimated source luminosity ( $L_{\mathrm{X}} \simeq 2 \times 10^{35} \mathrm{erg} \mathrm{s}^{-1}$ ) was fully consistent with the luminosity level detected by the previous observations, and the light curve showed no strong variability. Therefore, RX J1037.5-5647 is a long-period, low-luminosity Be/NS XBP that has been detected every time it has been observed. All these properties are typical of the persistent Be/NS pulsars, therefore Reig \& Roche (1999) suggest that RX J1037.5-5647 is a potential member of this class of sources.

\section{Observations and data reduction}

RX J1037.5-5647 was observed by XMM-Newton on 2009 January 9. The three EPIC cameras, i.e. one $p n$ (Strüder et al. 2001) and two MOS (Turner et al. 2001), were operated in small window mode, with a time resolution of $6 \mathrm{~ms}$ for the $p n$ camera and of $0.3 \mathrm{~s}$ for the two MOS cameras, and the medium thickness filter was used. The effective source exposure time was $\sim 28 \mathrm{ks}$ for the two MOS and $\sim 20 \mathrm{ks}$ for the $p n$, since the use of the small window mode for this camera implies a $\sim 30 \%$ dead time. Also the reflection grating spectrometer was used but, due to the source faintness, the count statistics was very low; therefore we ignored its spectra.

We used version 8.0 of the XMM-Newton Science Analysis System (SAS) to process the event files. After the standard pipeline processing, we looked for possible periods of high instrumental background, caused by flares of soft protons with energies less than a few hundred $\mathrm{keV}$. We found that the last $\sim 8 \mathrm{ks}$ of the observation were affected by a high soft-proton contamination, therefore we rejected the data of this part of the observation in the spectral analysis. The resulting effective exposure time was 14.5 and $20 \mathrm{ks}$ for the $p n$ and the MOS cameras, respectively.

\section{Timing analysis}

We selected source counts within a circular region around the source position, with extraction radii of 20 and $15^{\prime \prime}$ for the $p n$ and the two MOS cameras, respectively. Since in small window mode a very small sky area is imaged by the instrument, we were forced to select the corresponding background data on small circular areas, with radii of $20^{\prime \prime}$. We accumulated all the events with pattern range 0-4 (i.e. mono- and bi-pixel events) and 0-12 (i.e. from 1 to 4 pixel events) for the $p n$ and the two MOS cameras, respectively. This selection resulted in a net count rate $(\mathrm{CR})$ of $\sim 0.3 \mathrm{cts} \mathrm{s}^{-1}$ for the $p n$ camera and $\sim 0.13 \mathrm{cts} \mathrm{s}^{-1}$ for each of the two MOS cameras.

To measure the pulse period, we converted the event arrival times to the solar system barycenter, for each instrument separately; then, to increase the count statistic, we combined the three datasets and measured the pulse period by a standard

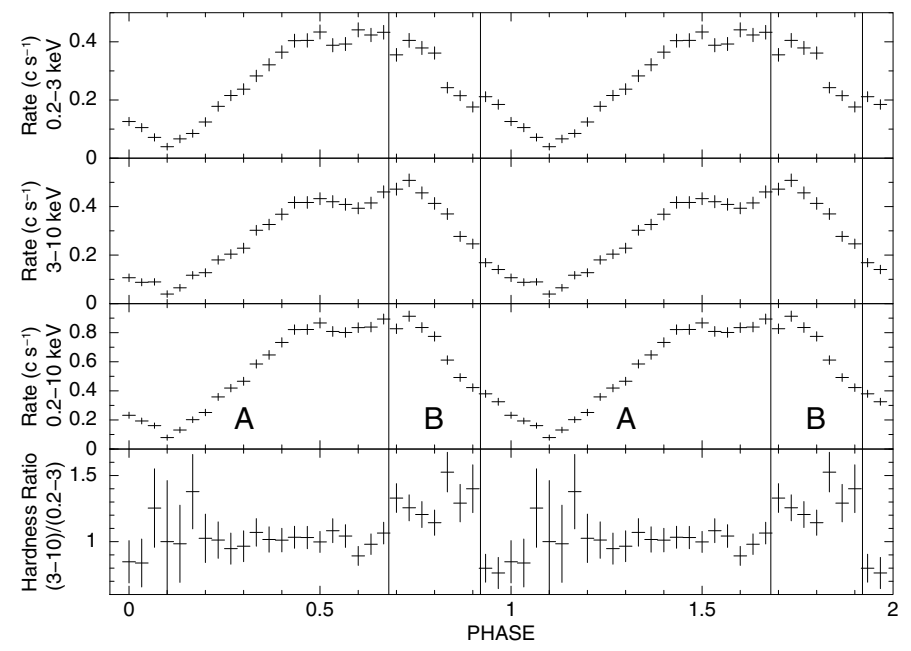

Fig. 1. Background-subtracted light curves of RX J1037.5-5647 in the energy ranges $0.2-3,3-10$, and $0.2-10 \mathrm{keV}$, folded at the best-fit period $P=853.4 \mathrm{~s}$. The vertical lines indicate the phase intervals used for the phase-resolved spectroscopy (see Sect. 5).

phase-fitting technique (Dall'Osso et al. 2003), obtaining a bestfit period $P=853.4 \pm 0.2 \mathrm{~s}$.

Thanks to the XMM-Newton observation, we could investigate the pulse profile, for the first time, even at energies below $3 \mathrm{keV}$, which could not be studied by RossiXTE. In Fig. 1 we show the folded light curves in the three energy intervals, 0.2-3 (soft), 3-10 (hard), and 0.2-10 keV, together with the folded hardness-ratio HR between the hard $(\mathrm{H})$ and soft $(\mathrm{S})$ light curves (computed as $\mathrm{H} / \mathrm{S}$ ). The shape of the pulse profile is similar in the three energy ranges. The measured pulsed fraction in the $0.2-10 \mathrm{keV}$ energy band, defined as $\left(\mathrm{CR}_{\max }-\mathrm{CR}_{\min }\right) /(2 \times$ $\left.\mathrm{CR}_{\text {average}}\right)$, is $75 \%$. The HR is rather constant along most of the pulse period, and it increases only at the end of the pulse maximum, when the source count rate starts to decrease.

It is interesting to compare the light curve of the whole $X M M-N e w t o n$ observation with the average one, which is obtained by assuming that all the pulses are equal to the average folded light curve. To this aim, we considered a binning time of $106.5 \mathrm{~s}$, corresponding to eight bins for each pulse period. In Fig. 2 we show the total $(p n+M O S 1+M O S 2)$ backgroundsubtracted light curve, in the $0.2-10 \mathrm{keV}$ energy range, and the average one, together with their difference. It shows that there are significant differences between the individual pulses, as well as some variability on longer timescales. Moreover, we checked that the source HR between the hard and soft bands is rather constant around the average value of $1.08\left(\chi_{v}^{2} /\right.$ d.o.f. $\left.=1.89 / 134\right)$ and does not depend on the source count rate (Fig. 3).

\section{Spectral analysis}

For the source and background spectra, we considered the same extraction parameters as we used for the light curves. We generated the applicable response matrices and ancillary files using the SAS tasks rmfgen and arfgen. To ensure the applicability of the $\chi^{2}$ statistics all spectra were rebinned with a minimum of 30 counts per bin and were fitted in the energy range $0.2-10 \mathrm{keV}$ using XSPEC 11.3.2. In the following, all spectral uncertainties and upper limits are given at $90 \%$ confidence level for one interesting parameter.

After checking that separate fits of the three cameras gave consistent results, we fitted them simultaneously to increase the 


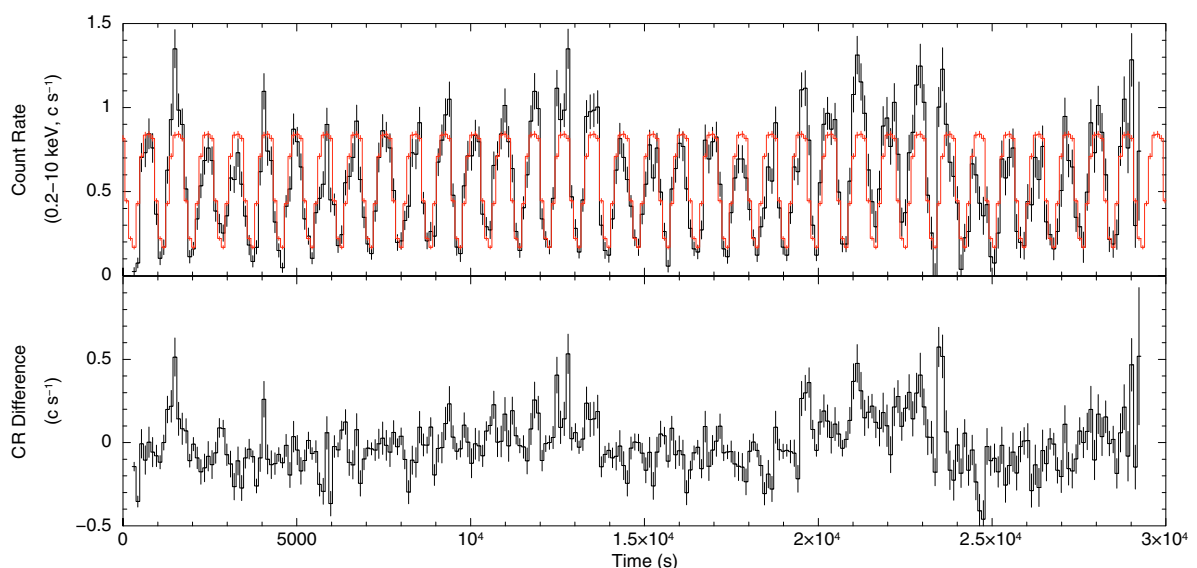

Fig. 2. Top: observed (black) and average (red) light curve of RX J1037.5-5647 in the energy range $0.2-10 \mathrm{keV}$. Bottom: count-rate difference between the individual time bins of the observed and average light curve.

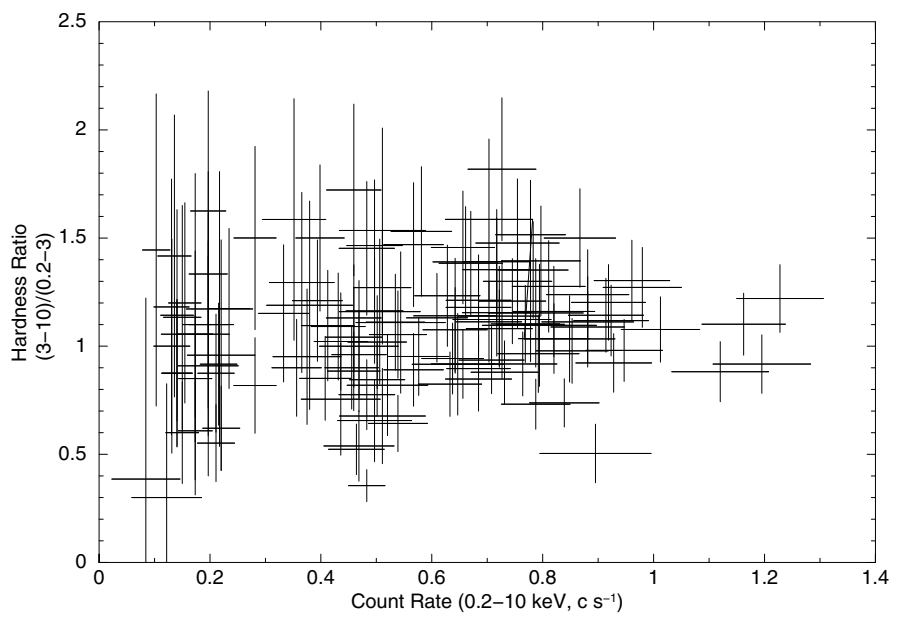

Fig. 3. Hardness-ratio variation of RX J1037.5-5647 as a function of the $0.2-10 \mathrm{keV}$ count rate, with a time bin of $213 \mathrm{~s}$.

statistics; to this aim, we introduced relative normalization factors among the spectra of the three cameras. Using an absorbed power-law $(P L)$ model, we obtained a hydrogen column density $N_{\mathrm{H}}=(6.2 \pm 0.5) \times 10^{21} \mathrm{~cm}^{-2}$ and a photon-index $\Gamma=0.94 \pm 0.03$, with $\chi_{v}^{2} /$ d.o.f. $=1.30 / 277$. On the other hand, using an absorbed blackbody $(B B)$ model, we obtained a hydrogen column density $N_{\mathrm{H}}=(0.9 \pm 0.2) \times 10^{21} \mathrm{~cm}^{-2}$ and a $B B$ temperature $k T_{\mathrm{BB}}=$ $1.52 \pm 0.03 \mathrm{keV}$, with $\chi_{v}^{2} /$ d.o.f. $=1.20 / 277$. Assuming a source distance of $5 \mathrm{kpc}$, we obtained a $B B$ radius $R_{\mathrm{BB}}=131 \pm 4 \mathrm{~m}$.

In both cases the fit of the spectrum with a single-component model shows large residuals, so we repeated the fit with a $P L+B B$ model. In this way we obtained a significant improvement in the fit quality (Fig. 4), since we obtained $\chi_{v}^{2} /$ d.o.f $=$ $0.88 / 275$. The corresponding best-fit parameters are $N_{\mathrm{H}}=(2.8 \pm$ $0.9) \times 10^{21} \mathrm{~cm}^{-2}, \Gamma=0.51_{-0.29}^{+0.17}$, and $k T_{\mathrm{BB}}=1.26_{-0.09}^{+0.16} \mathrm{keV}$. In comparison with the single $P L$ and $B B$ models, the F-test analysis provided a probability, respectively, of $P=1.26 \times$ $10^{-24}$ and $P=9.95 \times 10^{-20}$ that the improvement in the fit occurs by chance. Both components are significant at a $99 \%$ confidence level. The $P L$ normalization is $I_{\mathrm{PL}}=7.1_{-4.6}^{+4.5} \times$ $10^{-5} \mathrm{ph} \mathrm{cm}^{-2} \mathrm{~s}^{-1} \mathrm{keV}^{-1}$ at $1 \mathrm{keV}$ and, assuming a source distance of $5 \mathrm{kpc}$, we obtained a radius $R_{\mathrm{BB}}=128_{-21}^{+13} \mathrm{~m}$ for the $B B$ component. The unabsorbed flux in the energy range $0.2-$ $10 \mathrm{keV}$ is $f_{\mathrm{X}} \sim 4 \times 10^{-12} \mathrm{erg} \mathrm{cm}^{-2} \mathrm{~s}^{-1}$, about $42 \%$ of which is due to the $B B$ component. This translates into a source luminosity $L_{\mathrm{X}} \simeq 1.2 \times 10^{34} \mathrm{erg} \mathrm{s}^{-1}$.

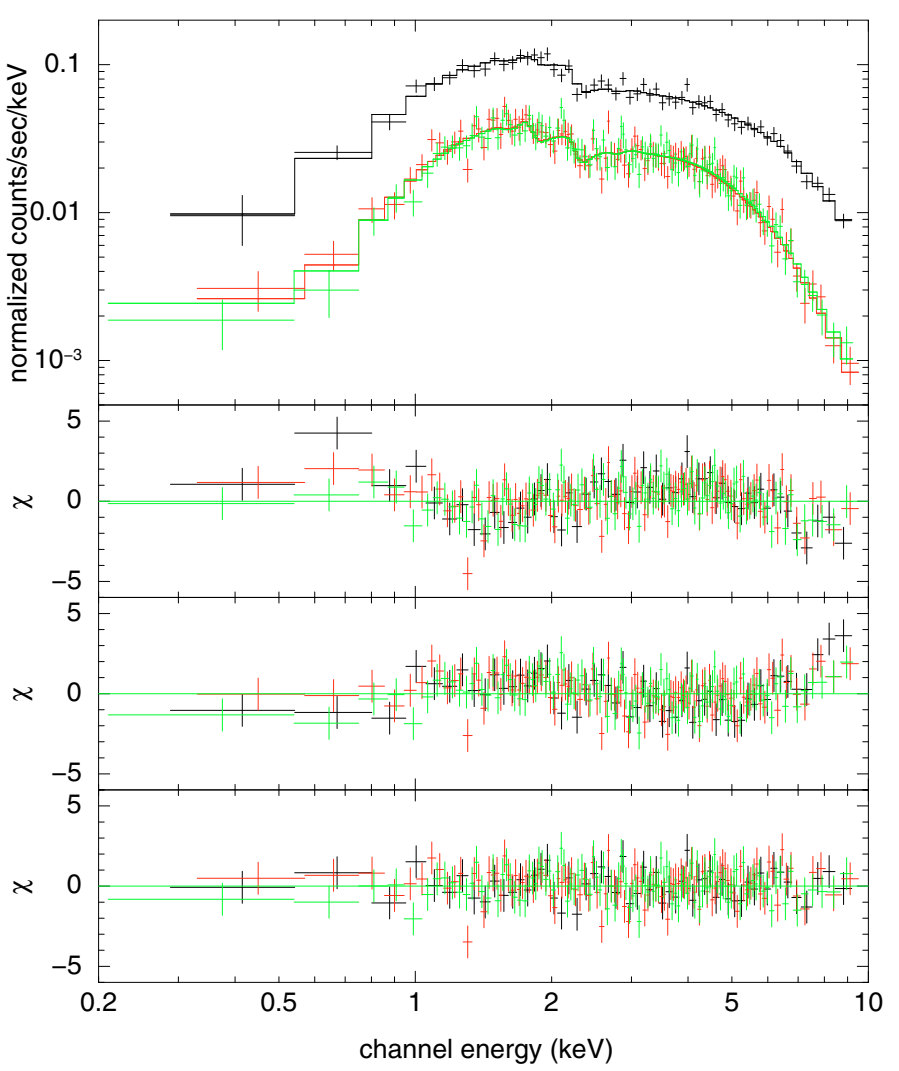

Fig. 4. Top panel: total spectrum of RX J1037.5-5647 with the best-fit $P L+B B$ model. The spectra of the $p n, M O S 1$ and MOS2 cameras are shown in black, red, and green, respectively. Middle panels: residuals (in units of $\sigma$ ) between data and model in the case of the single $P L$ and of the single $B B$. Bottom panel: residuals in the case of the $P L+B B$.

We also looked for narrow iron $\mathrm{K}_{\alpha}$ emission lines between 6 and $7 \mathrm{keV}$, with different widths between 0 and $0.5 \mathrm{keV}$. We found no evidence of such a component, with an upper limit on its equivalent width of $0.2 \mathrm{keV}$ (at $90 \%$ c.l.) at most.

\section{Phase-resolved spectroscopy}

In the source folded light curve (Fig. 1), we observed that the source spectrum becomes slightly harder at the end of the pulse maximum. To study the source behavior in more detail, we analyzed the background subtracted spectra in two different phase 
Table 1. Best-fit spectral parameters for the phase-resolved spectroscopy of RX J1037.5-5647, in the case of the independent fit of the two spectra.

\begin{tabular}{c|cc|cc|cc}
\hline \hline Phase interval & $\mathrm{A}$ & $\mathrm{B}$ & $\mathrm{A}$ & $\mathrm{B}$ & $\mathrm{A}$ & $\mathrm{B}$ \\
\hline$N_{\mathrm{H}}$ & $6.6 \pm 0.7$ & $6.6_{-1.3}^{+1.5}$ & $0.5 \pm 0.3$ & $0_{-0.0}^{+0.8}$ & $2.1_{-1.1}^{+1.4}$ & $0.9_{-0.8}^{+0.9}$ \\
\hline$\Gamma$ & $1.01 \pm 0.06$ & $0.79 \pm 0.10$ & & & $0.4_{-1.0}^{+0.5}$ & $-2.5_{-0.2}^{+0.3}$ \\
$f_{\mathrm{PL}}^{a}$ & $4.8(100 \%)$ & $5.5(100 \%)$ & & & $2.0(51 \%)$ & $1.5(31 \%)$ \\
\hline$k T_{\mathrm{BB}}(\mathrm{keV})$ & & & $1.45 \pm 0.03$ & $1.69_{-0.08}^{+0.06}$ & $1.22_{-0.09}^{+0.18}$ & $1.44_{-0.22}^{+0.12}$ \\
$R_{\mathrm{BB}}(\mathrm{m})$ & & & $139 \pm 6$ & $117_{-6}^{+9}$ & $142_{-24}^{+29}$ & $141_{-13}^{+11}$ \\
$f_{\mathrm{BB}}^{a}$ & & & $3.4(100 \%)$ & $4.1(100 \%)$ & $1.9(49 \%)$ & $3.4(69 \%)$ \\
\hline$f_{\mathrm{TOT}}^{a}$ & 4.8 & 5.5 & 3.4 & 4.1 & 3.9 & 4.9 \\
$\chi_{v}^{2} /$ d.o.f. & $2.00 / 80$ & $1.50 / 33$ & $1.45 / 80$ & $1.17 / 33$ & $1.05 / 78$ & $0.80 / 31$ \\
$P_{\mathrm{F}-\text { test }}(P L)$ & & & & & $4.79 \times 10^{-12}$ & $2.15 \times 10^{-5}$ \\
$P_{\mathrm{F}-\text { test }}(B B)$ & & & & & $1.33 \times 10^{-6}$ & $1.10 \times 10^{-3}$ \\
\hline
\end{tabular}

${ }^{a}$ Unabsorbed flux in the energy range $0.2-10 \mathrm{keV}$, in units of $10^{-12} \mathrm{erg} \mathrm{cm}^{-2} \mathrm{~s}^{-1}$.

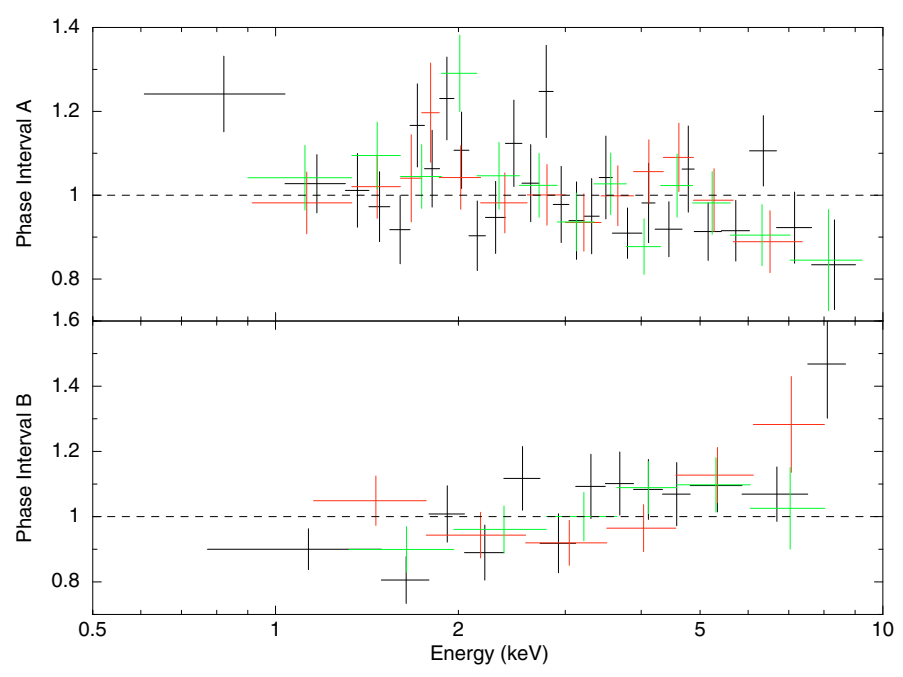

Fig. 5. Ratios of the spectra corresponding to the phase intervals shown in Fig. 1 to the average model spectrum. The parameters are fixed at the best-fit values for the total spectrum, except for the normalizations, which are at their best-fit values for each phase interval.

intervals, i.e. $\phi=0-0.68,0.92-1$ (phase interval A) and $\phi=$ 0.68-0.92 (phase interval B).

We fit the two background-subtracted spectra with the bestfit $P L+B B$ model of the phase-averaged spectrum, leaving only the relative normalization factors between the two phases free to vary, and we report the ratios of the two spectra of each instrument to this renormalized average model in Fig. 5. The data of the three detectors show that the spectra of phase intervals A and $\mathrm{B}$ are, respectively, softer and harder than the phase-averaged one, which implies a spectral variability along the pulse phase.

We then fitted the two spectra independently, using a single $P L$ model, a single $B B$ model, and a $P L+B B$ model; their best-fit parameters are reported in Table 1, where the hydrogen column density $N_{\mathrm{H}}$ is measured in units of $10^{21} \mathrm{~cm}^{-2}$, the radius $R_{\mathrm{BB}}$ of the $B B$ component (in metres) is obtained assuming a source distance of $5 \mathrm{kpc}$, and $P_{\mathrm{F}-\text { test }}(P L)$ and $P_{\mathrm{F}-\text { test }}(B B)$ are the probability that the improvement of the fit, compared to the single $P L$ and $B B$ model, respectively, occurs by chance. In both cases, we found that, as for the phase-averaged spectrum, the $B B$ model provides a better fit than the $P L$ model and that the use of a $P L+B B$ model improves significantly the spectral fit goodness, since there is a large decrease in the $\chi^{2}$ value, and the F-test
Table 2. Best-fit values for the black-body and power-law parameters, when the two spectra are fitted simultaneously with common values of $N_{\mathrm{H}}, \Gamma$, and $k T_{\mathrm{BB}}$ (case 1 ) or with common values of $N_{\mathrm{H}}, k T_{\mathrm{BB}}$, and $R_{\mathrm{BB}}$ (case 2).

\begin{tabular}{c|cc|cc}
\hline \hline Phase & \multicolumn{2}{|c}{ Case 1 } & \multicolumn{2}{c}{ Case 2 } \\
interval & $\mathrm{A}$ & $\mathrm{B}$ & $\mathrm{A}$ & $\mathrm{B}$ \\
\hline$N_{\mathrm{H}}$ & $1.4_{-0.5}^{+0.6}$ & $1.4_{-0.5}^{+0.6}$ & $2.3 \pm 1.0$ & $2.3 \pm 1.0$ \\
\hline$\Gamma$ & $-0.2_{-0.9}^{+0.3}$ & $-0.2_{-0.9}^{+0.3}$ & $0.51_{-0.57}^{+0.35}$ & $0.09_{-0.66}^{+0.37}$ \\
$I_{\mathrm{PL}}$ & $1.2_{-1.0}^{+2.0}$ & $2.4_{-2.0}^{+3.2}$ & $6.0_{-4.8}^{+5.1}$ & $4.3_{-3.2}^{+4.1}$ \\
$f_{\mathrm{PL}}^{a}$ & $1.5(39 \%)$ & $2.9(60 \%)$ & $2.0(51 \%)$ & $2.9(61 \%)$ \\
\hline$k T_{\mathrm{BB}}(\mathrm{keV})$ & $1.21_{-0.06}^{+0.07}$ & $1.21_{-0.06}^{+0.07}$ & $1.25_{-0.09}^{+0.13}$ & $1.25_{-0.09}^{+0.13}$ \\
$R_{\mathrm{BB}}(\mathrm{m})$ & $160_{-8}^{+11}$ & $147_{-19}^{+16}$ & $137_{-21}^{+13}$ & $137_{-21}^{+13}$ \\
$f_{\mathrm{BB}}^{a}$ & $2.3(61 \%)$ & $2.0(40 \%)$ & $1.9(49 \%)$ & $1.9(39 \%)$ \\
\hline$f_{\mathrm{TOT}}^{a}$ & 3.8 & 4.9 & 3.9 & 4.8 \\
$\chi_{\nu}^{2} /$ d.o.f. & \multicolumn{2}{|c|}{$0.99 / 115$} & \multicolumn{2}{c}{$0.96 / 115$} \\
\hline
\end{tabular}

a Unabsorbed flux in the energy range $0.2-10 \mathrm{keV}$, in units of $10^{-12} \mathrm{erg} \mathrm{cm}^{-2} \mathrm{~s}^{-1}$.

analysis gives a negligible probability that the improvement of the fit occurs by chance. Irrespective of the adopted model, we always found that the absorbing hydrogen column density is consistent with a constant value, as expected if it is due to the interstellar extinction. With both the single-component and the two-component models, the photon index shows evidence of variability between the two phase intervals, thus confirming the spectral variability; on the other hand, the $B B$ temperature and radius are only variable in the case of the single-component model, while they are rather constant in the case of the twocomponent model, since there is full agreement for the emission radii, and the temperatures are also comparable.

To investigate the relative variations of the two components with the period phase, we also simultaneously fitted the two spectra forcing the same value of $N_{\mathrm{H}}, \Gamma$, and $k T_{\mathrm{BB}}$ for the two phase intervals. In this case we obtained $N_{\mathrm{H}}=1.4_{-0.5}^{+0.6} \times$ $10^{21} \mathrm{~cm}^{-2}, \Gamma_{\mathrm{PL}}=-0.2_{-0.9}^{+0.3}$, and $k T_{\mathrm{BB}}=1.21_{-0.06}^{+0.07} \mathrm{keV}$, with $\chi_{v}^{2} /$ d.o.f. $=0.99 / 115$. The corresponding normalization values are reported in Table 2 , where the hydrogen column density $N_{\mathrm{H}}$ is measured in units of $10^{21} \mathrm{~cm}^{-2}$, the radius $R_{\mathrm{BB}}$ of the $B B$ component (in metres) is obtained assuming a source distance of $5 \mathrm{kpc}$, and the intensity $I_{\mathrm{PL}}$ of the $P L$ component is given in units of $10^{-5} \mathrm{ph} \mathrm{cm}^{-2} \mathrm{~s}^{-1} \mathrm{keV}^{-1}$ at $1 \mathrm{keV}$. With these constraints on the model parameters, the spectral changes as a function of 
the phase are reproduced by the variations in the relative contribution of the two components. The values reported in Table 2 show that almost all of the spectral variability comes from the $P L$ component, since its flux changes of a factor $\sim 2$ between the two phase intervals; on the other hand, there is only a $15 \%$ variation in the $B B$ flux, since we obtain comparable values for the emission radius. However, this finding is affected by large errors on the normalization of the $P L$ component.

As a further check, we modified the test model by linking the $B B$ parameters for the two spectra together, while both the photon-index $\Gamma$ and the normalization $I_{\mathrm{PL}}$ of the $P L$ component could vary independently in the two phase intervals. The resulting best fit gives $N_{\mathrm{H}}=(2.3 \pm 1.0) \times 10^{21} \mathrm{~cm}^{-2}$, $k T_{\mathrm{BB}}=1.25_{-0.09}^{+0.13} \mathrm{keV}$, and $R_{\mathrm{BB}}=137_{-21}^{+13} \mathrm{~m}$ (for a source distance of $5 \mathrm{kpc}$ ). The power-law parameters are shown in Table 2. Even with this model we found a very good fit quality $\left(\chi_{v}^{2} /\right.$ d.o.f. $=$ $0.96 / 115)$, fully comparable to the previous case. This result suggests that it is possible to attribute the whole spectral variability to the $P L$ component. However, we note that the power-law parameters are not well-constrained, since they are always affected by large errors. Moreover, for the spectrum of the phase interval $\mathrm{B}$ the independent fit with a $P L+B B$ model provides a large negative value of $\Gamma$, which is very different to what is obtained from any other fit. This result can be explained by the large contribution of the $B B$ component to the total flux, while in the other cases most of the flux is due to the $P L$ component: in fact, for the phase interval $B$ we can also obtain a good fit using a simple $B B$ model $\left(\chi_{v}^{2} /\right.$ d.o.f. $\left.=1.17 / 33\right)$. This means that an additional $P L$ component is only required to fit the high-energy end of the spectrum, thus providing a high negative value of the photon index.

\section{Discussion}

Before the XMM-Newton observation of RX J1037.5-5647, the only X-ray investigation of this source was performed by RossiXTE (Reig \& Roche 1999). Therefore it is interesting to compare our results with those obtained ten years before.

From the timing analysis, we obtained a new refined pulse period, $P=853.4 \pm 0.2 \mathrm{~s}$, which is lower than the previous value of $860 \pm 2 \mathrm{~s}$ found by RossiXTE. This implies a pulsar spin-up during the past ten years, with $\dot{P}=(-1.91 \pm 0.58) \times 10^{-8} \mathrm{~s} \mathrm{~s}^{-1}$, and suggests a momentum transfer to the neutron star.

Based on the observed source flux, we estimated a source luminosity $L_{\mathrm{X}} \sim 10^{34} \mathrm{erg} \mathrm{s}^{-1}$ in the $2-10 \mathrm{keV}$ energy range (assuming a source distance of $5 \mathrm{kpc}$ ), which is one order of magnitude lower than the luminosity level detected by RossiXTE. From the spectral analysis, we obtained a hydrogen column density $N_{\mathrm{H}}=(2.8 \pm 0.9) \times 10^{21} \mathrm{~cm}^{-2}$, which is much lower than the values estimated by RossiXTE: the lowest result (obtained with a double BB model) was $(2.1 \pm 0.3) \times 10^{22} \mathrm{~cm}^{-2}$, which is almost one order of magnitude higher than our result; moreover, using our same $P L+B B$ model, the RossiXTE data provided a best-fit value of $(8.2 \pm 0.5) \times 10^{22} \mathrm{~cm}^{-2}$, which is $\sim 30$ times higher than our value. On this subject, we emphasize that the energy range of the RossiXTE spectral analysis (above $3 \mathrm{keV}$ ) is not wellsuited to a good estimate of $N_{\mathrm{H}}$, while the low-energy end of the XMM-Newton spectra $(0.2 \mathrm{keV})$ allows a much more reliable analysis. Moreover, we also note that LS 1698, the optical counterpart of RX J1037.5-5647, has a color excess $E(B-V) \simeq 0.75$ (Motch et al. 1997). Assuming $A_{\mathrm{V}}=3.1 E(B-V)$ and the average relation $A_{\mathrm{V}}=N_{\mathrm{H}} \times 5.59 \times 10^{-22} \mathrm{~cm}^{-2}$ between optical extinction and X-ray absorption (Predehl \& Schmitt 1995), this would predict $N_{\mathrm{H}}=4.16 \times 10^{21} \mathrm{~cm}^{-2}$, a value comparable to our result. This indicates that in the XMM-Newton observation the measured absorption stems from the interstellar medium and not from local matter around the system, which has a negligible effect, while in the RossiXTE observations intrinsic absorption is also possible.

The RossiXTE spectrum analyzed by Reig \& Roche (1999) was fitted with the same model $(P L+B B)$, resulting in a much steeper power-law component $(\Gamma=1.84 \pm 0.06$ instead of $\left.0.51_{-0.29}^{+0.17}\right)$ and in a blackbody component with a much higher temperature $\left(k T=2.9 \pm 0.2 \mathrm{keV}\right.$ instead of $\left.k T=1.26_{-0.09}^{+0.16} \mathrm{keV}\right)$. Even in this case, it is possible that these differences arise not only because the spectral analysis was performed in two different energy ranges, but also because the source was one order of magnitude brighter during the RossiXTE observation. On the other hand, the two analyses provided comparable values for the emission radius of the blackbody component $(100 \pm 10 \mathrm{~m}$ for RossiXTE and $128_{-21}^{+13} \mathrm{~m}$ for XMM-Newton).

We found no evidence of an Fe-K line in the source spectrum, with an upper limit of $0.2 \mathrm{keV}$ to its equivalent width (at $90 \%$ c.1.), while Reig \& Roche (1999) report a Gaussian line at $E=6.5 \pm 0.2 \mathrm{keV}$, with an equivalent width $\simeq 65 \mathrm{eV}$. It is very likely that XMM-Newton missed a clear detection of this feature because of the different luminosity level of the source, since a lower accretion rate on the NS implies a smaller amount of material around it and, then, a reduced probability for the emerging $\mathrm{X}$-rays to produce an iron line feature.

The comparison of the pulse profiles in two energy bands suggests a possible spectral variability of the source with the pulse phase, so we performed a phase-resolved spectral analysis, which previously had never been carried out for this source. The fit of two phase-resolved spectra with the best-fit model of the phase-averaged one confirmed the suspected variability. The independent fit of these spectra with different emission models showed that the absorbing hydrogen column density is nearly constant along the pulse phase, as expected if it is due to the interstellar extinction; therefore the observed variability must be due to the intrinsic source emission. The same fit proved also that, for both spectra, both the thermal and non-thermal component are needed to reproduce the observed data. Finally, the simultaneous fit of the two spectra with a common value of $N_{\mathrm{H}}$ shows that the whole spectral variability can be attributed to the $P L$ component, since the $B B$ one is nearly constant along the pulse.

Reig \& Roche (1999) suggest that RX J1037.5-5647 is a persistent Be binary pulsars, since this source has been detected every time it has been observed and does not show large outbursts. To investigate the long-term evolution of RX J1037.5-5647, we considered all the X-ray flux measurements obtained for this source since its discovery in the early 70s. For the UHURU (Forman et al. 1978), OSO 7 (Markert et al. 1979) and ARIEL V (Warwick et al. 1981) detections, we used the published CR values and the appropriate count-rate-to-flux conversion factors to obtain the corresponding $2-10 \mathrm{keV}$ fluxes. In the case of the RossiXTE All Sky Monitor (ASM), we considered the day-by-day light curve, which covers the time interval from January 1996 to March 2009. Since the detection sensitivity of this instrument is very low, in several cases it failed to detect RX J1037.5-5647, owing to its low flux. For this reason and for estimating the average flux level of the source, we binned the data in five different time bins of 1000 days each. We calculated the average CR in each bin and, assuming our best-fit $P L+B B$ emission model, we used the HEASARC tool WebPIMMS to obtain the corresponding average flux between 2 and $10 \mathrm{keV}$. On the other hand, we considered the results obtained by Reig \& Roche (1999) to calculate 


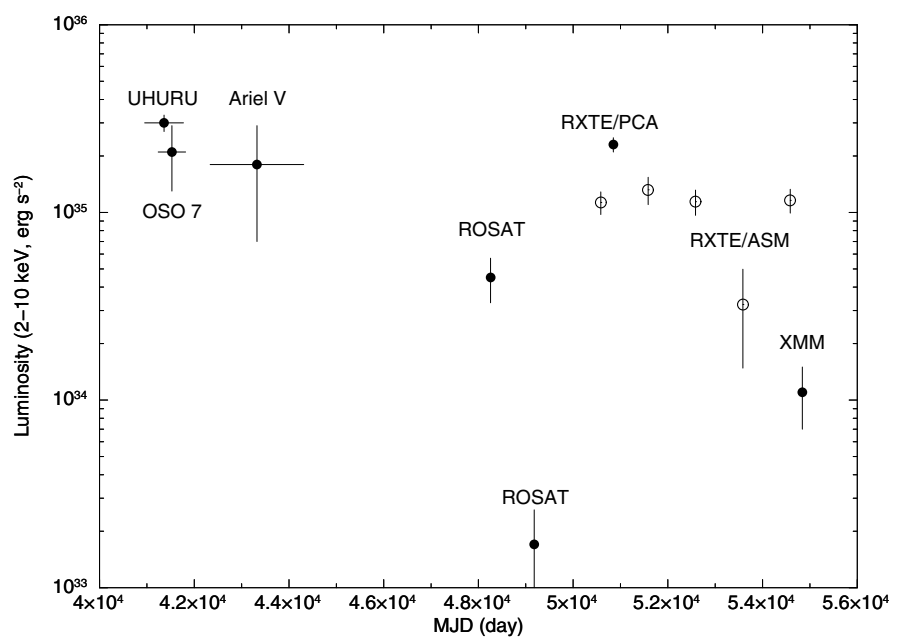

Fig. 6. Luminosity history of RX J1037.5-5647 from 1971 to 2009. Reported luminosities are based on the unabsorbed flux in the $2-10 \mathrm{keV}$ and on a source distance of $5 \mathrm{kpc}$ (Motch et al. 1997). Open circles refer to the average luminosity (between 2 and $10 \mathrm{keV}$ ) estimated by RossiXTE ASM in five time intervals of 1000 days each.

the source flux (in the energy range $2-10 \mathrm{keV}$ ) measured by the RossiXTE Photon Counting Array (PCA). Finally, we also considered the CRs measured by ROSAT, which were obtained during the All Sky Survey (Voges et al. 1999) and in a subsequent, specific observation (Motch et al. 1997). In their case, we had to infer the source flux in the $2-10 \mathrm{keV}$ energy band from the CR measured in the $0.1-2.4 \mathrm{keV}$ range. To do this, we again used the WebPIMMS tool and assumed our best-fit $P L+B B$ emission model.

Assuming a source distance of $5 \mathrm{kpc}$ (Motch et al. 1997), we used the calculated fluxes to estimate the corresponding luminosities, which are reported in Fig. 6. It shows that, in most observations performed before XMM-Newton, RX J1037.5-5647 was detected at a luminosity level $L_{\mathrm{X}}=(1-3) \times 10^{35} \mathrm{erg} \mathrm{s}^{-1}$ between 2 and $10 \mathrm{keV}$, and that the average luminosity observed by the $A S M$ is also $\sim 10^{35} \mathrm{erg} \mathrm{s}^{-1}$. This behavior is remarkable, since the performed observations span a time interval of about 35 years, and confirms that RX J1037.5-5647 is a persistent, low-luminosity binary pulsar. However, the observation performed by XMM-Newton detected the source at a luminosity level $L_{X} \sim 10^{34} \mathrm{erg} \mathrm{s}^{-1}$, which is one order of magnitude lower than the previous average value. Moreover, it was detected with an even lower luminosity in one of the ROSAT observations. It is possible that the ROSAT results stem from an inaccurate estimate of the source flux, since it is obtained from a CR measurement in a different energy band, but even such an error could hardly explain the very low luminosity value obtained in the second ROSAT observation. These results confirm that RX J1037.55647 is a persistent source, since it has been detected every time it has been observed, but show also that the source is characterized by a significant variability.

A $B B$ excess above the main power-law component has also been detected in the case of LS I +61 ${ }^{\circ} 235 / \mathrm{RX} \mathrm{J0146.9+6121}$ (La Palombara \& Mereghetti 2006) and X Persei/4U 0352+309 (Coburn et al. 2001; La Palombara \& Mereghetti 2007), which are the two main members of the class of the persistent Be/NS XBPs. A similar feature was also observed during the lowluminousity quiescent state of the transient Be/NS XBP 3A 0535+262 (Orlandini et al. 2004; Mukherjee \& Paul 2005), which is comparable to the previous persistent Be pulsars for

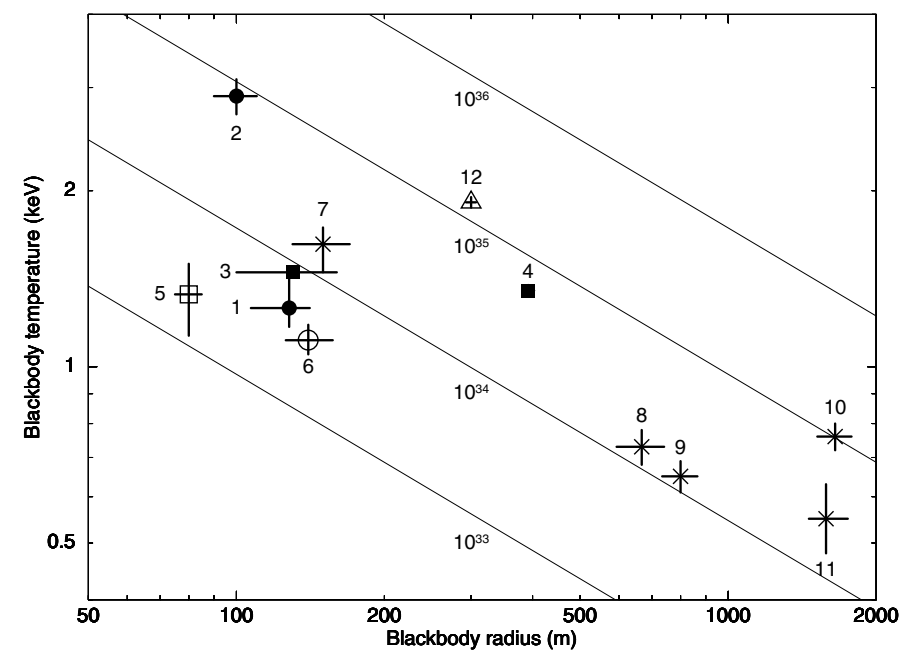

Fig. 7. Best-fit values for radius and temperature of the $B B$ component in the case of RX J1037.5-5647 (filled circles), 4U 0352+309 (filled squares), RX J0146.9+6121 (open circle), 3A 0535+262 (open square), SAX J2103.5+4545 (open triangle), and 4U 2206+54 (crosses). The continuous lines connect the blackbody parameters corresponding to four different levels of luminosity (in erg s ${ }^{-1}$ ). References: 1 - this work; 2 - Reig \& Roche (1999); 3 - Coburn et al. (2001); 4 - La Palombara \& Mereghetti (2007); 5 - Mukherjee \& Paul (2005); 6 - La Palombara \& Mereghetti (2006); 7 - Masetti et al. (2004); 8, 9, 10 - Torrejón et al. (2004); 11 - Reig et al. (2009); 12 - İnam et al. (2004).

its low luminosity $\left(L_{\mathrm{X}} \leq 10^{35} \mathrm{erg} \mathrm{s}^{-1}\right)$ and long pulse period $(P=103$ s). A blackbody component has also been observed in 4U 2206+54 (Masetti et al. 2004; Torrejón et al. 2004; Reig et al. 2009), a high-mass X-ray binary with a comparable luminosity and a very long pulse period $(P=5560 \mathrm{~s})$, and in the X-ray pulsar $(P=358.6 \mathrm{~s})$ SAX J2103.5+4545 (İnam et al. 2004). In Fig. 7 we report the best-fit radius and temperature for these sources, together with lines showing four different levels of the blackbody luminosity. In the case of RX J1037.55647 and $4 \mathrm{U} 0352+309$, we report two set of values, since both sources were observed by RossiXTE and XMM-Newton at two different luminosity levels $\left(L_{X} \sim 10^{34}\right.$ and $\sim 10^{35} \mathrm{erg} \mathrm{s}^{-1}$, respectively): in the case of RX J1037.5-5647 the low and high luminosity were observed by XMM-Newton and RossiXTE respectively, while for $4 \mathrm{U} 0352+309$ it was the opposite. We report various measurements also for $4 \mathrm{U} 2206+54$, corresponding to different observations. The figure shows that the results obtained for RX J1037.5-5647 are in full agreement with those obtained by various observations of the previous sources, since their spectral parameters are clustered in a narrow range of values, i.e. $k T_{\mathrm{BB}} \sim 1-1.5 \mathrm{keV}$ and $R_{\mathrm{BB}} \sim 100 \mathrm{~m}$. We emphasize that, in all these cases, the estimated total source X-ray luminosity is $\sim 10^{34} \mathrm{erg} \mathrm{s}^{-1}$, with a $20-40 \%$ contribution of the blackbody component. For the persistent Be pulsars RX J1037.55647 and $4 \mathrm{U} 0352+309$, spectral values outside the previous range (points 2 and 4) were obtained by the XMM-Newton observation of $4 \mathrm{U} 0352+309$ and the RossiXTE observation of RX J1037.5-5647, when the sources were detected at a higher luminosity level $\left(L_{X} \sim 10^{35} \mathrm{erg} \mathrm{s}^{-1}\right)$; however, we note that comparable blackbody parameters were also obtained for SAX $\mathrm{J} 2103.5+4545$, which is characterized by a comparable luminosity. On the other hand, in most cases the non-Be binary pulsar $4 \mathrm{U} 2206+54$ also shows a different behavior when observed at a comparable luminosity level: $k T_{\mathrm{BB}}<1 \mathrm{keV}$ and $R_{\mathrm{BB}}>500 \mathrm{~m}$ (points 8 to 11). Finally, we note that the XMM-Newton spectrum 


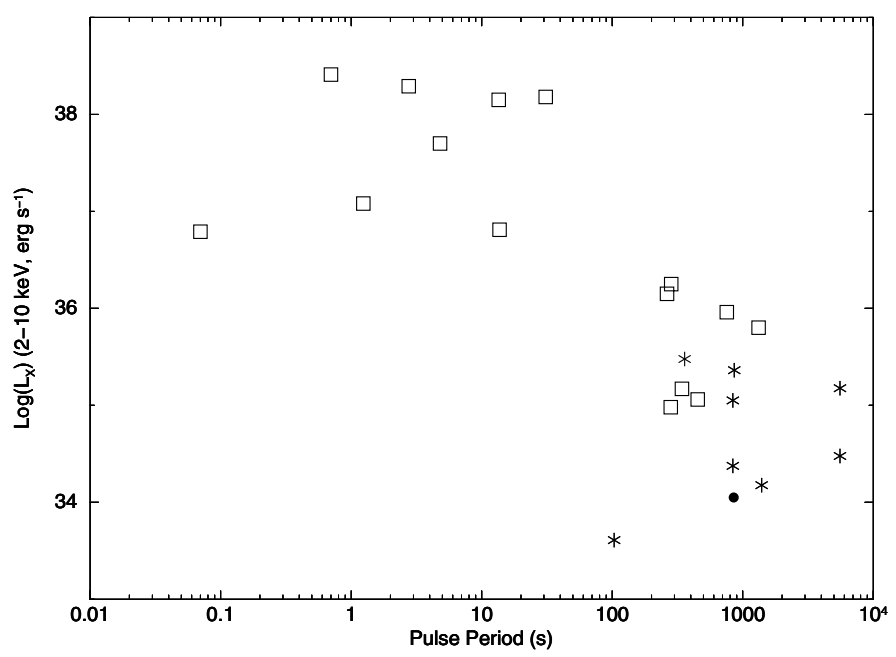

Fig. 8. X-ray luminosity (in the $2-10 \mathrm{keV}$ energy range) of the XBPs with a detected thermal excess as a function of the pulse period. The filled circle refers to the XMM-Newton observation of RX J1037.55647 , asterisks refer to other detections of hot BB pulsars, open squares to all the other bright sources.

of RX J1037.5-5647 is characterized by the hardest $P L$ component among the previous sources, since it has a photon-index $\Gamma=0.5$, while $\Gamma \geq 1$ in all the other sources.

An X-ray excess has also been observed in several other XBPs (see La Palombara \& Mereghetti 2006, for a review) but, in contrast to the previous sources, in their case the fit of this excess with a thermal emission model provided low temperatures $(k T<0.5 \mathrm{keV})$ and large emitting regions $(R>100 \mathrm{~km})$; for this reason, this feature is usually described as a soft excess. In Fig. 8 we report the luminosity and pulse period of the XBPs with a detected thermal excess. They are divided into two distinct groups: the sources in the first group are characterized by high luminosity $\left(L_{\mathrm{X}} \geq 10^{37} \mathrm{erg} \mathrm{s}^{-1}\right)$ and short pulse period $(P<$ $100 \mathrm{~s}$ ), and in most cases they are in close binary systems with an accretion disk; those in the second group have low luminosities $\left(L_{\mathrm{X}} \leq 10^{36} \mathrm{erg} \mathrm{s}^{-1}\right)$ and long pulse periods $(P>100 \mathrm{~s})$, since they have wide orbits and are wind-fed systems. Among the sources of the second group of XBPs, the six pulsars discussed in the previous paragraph (reported as asterisks and filled circle in Fig. 8) are the ones that have, at the same time, the lowest luminosities and the longest periods with the only exception of SAX J2103.5+4545, which has the highest luminosity of this group of sources. They are characterized by a $B B$ component with a high temperature and a small emission radius, while the others show soft thermal excesses with low temperature $(k T<$ $0.5 \mathrm{keV})$ and large emission area $(R \sim$ a few hundred $\mathrm{km})$. The hot $B B$ spectral component separates these low-luminosity and long-period sources from all the other pulsars, strongly suggesting that they form a distinct and well-defined class of binary pulsars.

Based on our results and on the emission models proposed by Hickox et al. (2004), the luminosity of RX J1037.5-5647 is too small to interpret the soft excess in terms of reprocessing of the hard X-ray photons in optically thick accreting material. Moreover, both the thermal emission and the hard X-ray reprocessing in a diffuse, optically thin gas around the neutron star are unlikely, since these processes would not give a blackbody spectrum. We therefore favor interpreting the soft excess in RX J1037.5-5647 as thermal emission from the neutron star polar cap. If we assume that the source is in the "accretor" status, with matter accretion on the NS surface, the blackbody emitting radius of $\sim 130 \mathrm{~m}$ is consistent with the expected size of the polar cap. In fact, assuming $M_{\mathrm{NS}}=1.4 M_{\odot}$ and $R_{\mathrm{NS}}=10^{6} \mathrm{~cm}$, the source luminosity $L_{\mathrm{X}}=1.2 \times 10^{34} \mathrm{erg} \mathrm{s}^{-1}$ implies an accretion rate $\dot{M} \simeq 6.4 \times 10^{13} \mathrm{~g} \mathrm{~s}^{-1}$ and, adopting $B_{\mathrm{NS}}=10^{12} \mathrm{G}$, a magnetospheric radius $R_{\mathrm{m}} \simeq 2.5 \times 10^{9} \mathrm{~cm}$ (Campana et al. 1998). In this case, based on the relation $R_{\mathrm{col}} \sim R_{\mathrm{NS}}\left(R_{\mathrm{NS}} / R_{\mathrm{m}}\right)^{0.5}$ (Hickox et al. 2004), we would obtain $R_{\text {col }} \sim 200 \mathrm{~m}$, comparable to the estimated blackbody emitting radius

The above results suggest that the observed thermal component can be attributed to the emission from the NS polar caps, as already proposed for 4U $0352+309$, RX J0146.9+6121, and $3 \mathrm{~A} 0535+262$. If this description is correct, we would expect to observe some variability of the thermal component along the pulse phase. This hypothesis cannot be confirmed by the present data, since the phase-resolved spectroscopy shows that the significant spectral variability detected along the pulse phase can come from the $P L$ component, with no variation of the $B B$ one.

\section{Conclusions}

We have reported the analysis of the data collected by XMMNewton in a $\sim 28 \mathrm{ks}$ observation of the Be/NS X-ray pulsar RX J1037.5-5647. The unabsorbed flux corresponds to a source luminosity $L_{\mathrm{X}} \simeq 1.2 \times 10^{34} \mathrm{erg} \mathrm{s}^{-1}$ in the $0.2-10 \mathrm{keV}$ energy range, which is about one order of magnitude lower than the average level of the previous observations. The source pulse period is $P=853.4 \pm 0.2 \mathrm{~s}$, which, together with previous measurements, implies an average pulsar spin-up $\dot{P} \simeq-2 \times 10^{-8} \mathrm{~s} \mathrm{~s}^{-1}$ in the past decade.

We were able to perform the first accurate timing and spectral analysis below $2 \mathrm{keV}$ for this source. The pulse profile shows a complex structure, which is not sinusoidal but does not depend on energy. In the source spectrum we detected a count excess above the main power-law component, described by a blackbody with $k T_{\mathrm{BB}} \simeq 1.3 \mathrm{keV}$ and $R_{\mathrm{BB}} \simeq 130 \mathrm{~m}$, which contributes $\sim 40 \%$ to the total source luminosity. We found no evidence of a narrow iron $\mathrm{K}_{\alpha}$ line between 6 and $7 \mathrm{keV}$, with an upper limit of $0.2 \mathrm{keV}$ on its equivalent width.

The new XMM-Newton detection of the long-period Be binary pulsar RX J1037.5-5647 at a low luminosity level confirms that it belongs to the class of the persistent Be binary pulsars, whose main representatives are $4 \mathrm{U} 0352+309$ and RX $\mathrm{J} 0146.9+6121$. Moreover, there is full agreement among the results of the spectral analysis of these three sources; therefore it reinforces the hypothesis that a thermal excess of blackbody type, with high temperature $(k T>1 \mathrm{keV})$ and small emission area $(R<0.5 \mathrm{~km})$, is a common feature of this type of source. However, this type of excess is not exclusive of the persistent low-luminosity Be binary pulsars, since the transient source $3 \mathrm{~A}$ $0535+262$ also shows this component. This means that, on one hand, all the low-luminosity Be X-ray binary pulsars show this component but, on the other, the presence of such component in a Be binary does not imply that the system is a persistent lowluminosity source.

As in the other two sources, also for RX J1037.5-5647 the blackbody radius is comparable to the estimated size of the NS polar-cap, which suggests that the origin of this component is on the NS surface. However, the phase-resolved spectroscopy does not confirm this scenario, since the observed spectral variability along the pulse period can be attributed to the power-law component and is consistent with a constant thermal component.

Acknowledgements. This work is based on observations obtained with $X M M-N e w t o n$, an ESA science mission with instruments and contributions 
directly funded by ESA Member States and NASA. The XMM-Newton data analysis is supported by the Italian Space Agency (ASI/INAF contract I/088/06/0). P.E. thanks the Osio Sotto City Council for support with a "G. Petrocchi" fellowship.

\section{References}

Burderi, L., Di Salvo, T., Robba, N. R., La Barbera, A., \& Guainazzi, M. 2000, ApJ, 530, 429

Campana, S., Colpi, M., Mereghetti, S., Stella, L., \& Tavani, M. 1998, A\&ARv, 8,279

Coburn, W., Heindl, W. A., Gruber, D. E., et al. 2001, ApJ, 552, 738

Dall'Osso, S., Israel, G. L., Stella, L., Possenti, A., \& Perozzi, E. 2003, ApJ, 599,485

Delgado-Martí, H., Levine, A. M., Pfahl, E., \& Rappaport, S. A. 2001, ApJ, 546, 455

Eger, P., \& Haberl, F. 2008, A\&A, 491, 841

Forman, W., Jones, C., Cominsky, L., et al. 1978, ApJS, 38, 357

Haberl, F., \& Pietsch, W. 2004, A\&A, 414, 667

Haberl, F., \& Pietsch, W. 2005, A\&A, 438, 211

Haberl, F., \& Sasaki, M. 2000, A\&A, 359, 573

Haberl, F., Eger, P., \& Pietsch, W. 2008, A\&A, 489, 327

Hickox, R. C., Narayan, R., \& Kallman, T. R. 2004, ApJ, 614, 881

İnam, S. Ç., Baykal, A., Swank, J., \& Stark, M. J. 2004, ApJ, 616, 463

Israel, G. L., Campana, S., Covino, S., et al. 2000, ApJ, 531, L131

Kohno, M., Yokogawa, J., \& Koyama, K. 2000, PASJ, 52, 299

La Palombara, N., \& Mereghetti, S. 2006, A\&A, 455, 283

La Palombara, N., \& Mereghetti, S. 2007, A\&A, 474, 137

Macomb, D. J., Fox, D. W., Lamb, R. C., \& Prince, T. A. 2003, ApJ, 584, L79

Majid, W. A., Lamb, R. C., \& Macomb, D. J. 2004, ApJ, 609, 133
Manousakis, A., Walter, R., Audard, M., \& Lanz, T. 2009, A\&A, 498, 217

Markert, T. H., Laird, F. N., Clark, G. W., et al. 1979, ApJS, 39, 573

Masetti, N., Dal Fiume, D., Amati, L., et al. 2004, A\&A, 423, 311

Motch, C., Haberl, F., Dennerl, K., Pakull, M., \& Janot-Pacheco, E. 1997, A\&A, 323,853

Mukherjee, U., \& Paul, B. 2005, A\&A, 431, 667

Nagase, F. 2002, J. ApA, 23, 59

Naik, S., \& Paul, B. 2004, ApJ, 600, 351

Negueruela, I. 1998, A\&A, 338, 505

Orlandini, M., Bartolini, C., Campana, S., et al. 2004, Nucl. Phys. B Proc. Suppl., 132,476

Paul, B., Nagase, F., Endo, T., et al. 2002, ApJ, 579, 411

Predehl, P., \& Schmitt, J. H. M. M. 1995, A\&A, 293, 889

Ramsay, G., Zane, S., Jimenez-Garate, M. A., den Herder, J.-W., \& Hailey, C. J. 2002, MNRAS, 337, 1185

Reig, P., \& Roche, P. 1999, MNRAS, 306, 100

Reig, P., Torrejón, J. M., Negueruela, I., et al. 2009, A\&A, 494, 1073

Sasaki, M., Pietsch, W., \& Haberl, F. 2003, A\&A, 403, 901

Strüder, L., Briel, U., Dennerl, K., et al. 2001, A\&A, 365, L18

Torrejón, J. M., Kreykenbohm, I., Orr, A., Titarchuk, L., \& Negueruela, I. 2004, A\&A, 423, 301

Turner, M. J. L., Abbey, A., Arnaud, M., et al. 2001, A\&A, 365, L27

Ueno, M., Yamaguchi, H., Takagi, S.-I., Yokogawa, J., \& Koyama, K. 2004, PASJ, 56, 175

Voges, W., Aschenbach, B., Boller, T., et al. 1999, A\&A, 349, 389

Warwick, R. S., Marshall, N., Fraser, G. W., et al. 1981, MNRAS, 197, 865

Yokogawa, J., Imanishi, K., Ueno, M., \& Koyama, K. 2000a, PASJ, 52, L73

Yokogawa, J., Paul, B., Ozaki, M., et al. 2000b, ApJ, 539, 191

Yokogawa, J., Imanishi, K., Tsujimoto, M., Koyama, K., \& Nishiuchi, M. 2003, PASJ, 55, 161 